\title{
Sudden death due to a colloid cyst of the third ventricle: case report
}

AN Vadysinghe*, CD Wickramsinghe

Department of Forensic Medicine, Faculty of Medicine, University of Peradeniya

*Corresponding author: Tel:0718040320. E-mail address: amal_vadysinghe@yahoo.com

\begin{abstract}
Colloid cyst of the third ventricle is a benign tumour arising in the anterior roof of the third ventricle in close proximity to the foramen of Monro. It occurs in 3 persons per million per year and ranges in presentation from episodic headache to sudden death. We describe a rare case of sudden death due to a colloid cyst in the third ventricle which has not been reported in the local literature. A 15 year old girl suffering from episodic headaches being treated as migraine for the past 3 years developed progressive headaches of acute onset. She died about 5 hours after admission to the peripheral hospital. An autopsy revealed severe cerebral oedema and dissection of the fixed brain revealed a colloid cyst in the third ventricle with a dilated ventricular system. Cause of death was given as colloid cyst of the third ventricle. This case report shows that although rare it is important for both the Clinician and the Forensic Pathologist to include this entity in the differential diagnosis of headaches in children and in hydrocephalus detected at autopsy. Demonstration of the colloid cyst is difficult as it is easily missed in dissection which signifies the importance of brain fixation.
\end{abstract}

Keywords: Headache, sudden unexpected death, colloid cyst

\section{Introduction}

Brain tumours are important for forensic pathologists as a cause of sudden death and we report such a case due to a colloid cyst in the third ventricle. This is a rare benign tumour arising in the anterior roof of the third ventricle at the level of the foramen of Monro occurring in 3 persons per million per year[1]. It accounts for $1 \%$ of all primary brain tumours and $15-20 \%$ of all intraventricular masses. This is the commonest lesion occurring in the anterior roof of the third ventricle[2]. Important differential diagnoses are ependymomas and meningiomas.

\section{Case report}

A 15 year old girl had been suffering from episodic headaches for the past 3 years. She had intermittently taken treatment as for migraine by a general practitioner. She presented to the peripheral hospital with a progressive severe headache and vomiting. On examination she was drowsy and had no neurological signs until later she developed generalized convulsions. The patient had a cardio-respiratory arrest and resuscitation had failed. Death was confirmed 5 hours after admission. She had been vaccinated with rubella two weeks before death. During that time there were several reported incidents of reactions to the vaccine. She had no significant medical conditions or family history of epilepsy or other sudden unexplained deaths.

Autopsy findings: Medico-legal autopsy was performed approximately 20 hours after death. The body was that of a 15 year old female 150 $\mathrm{cm}$ in length, $48 \mathrm{~kg}$ in weight. No external 
evidence of violence was identified, and there were no external signs of natural disease or congenital abnormalities. The brain showed severe cerebral oedema (fig. 1 and 2). Dissection of the fixed brain revealed a colloid cyst of $2.0 \times 1.5 \times 1.5 \mathrm{~cm}$ size in the third ventricle with a dilated ventricular system (fig. 3 and 4). The lungs showed moderate pulmonary oedema. Histology of the brain showed oedema and hypoxic changes. Examination of other systems and histology of rest of the organs appeared normal. Virological studies including Rubella were reported negative. I concluded that death was due to colloid cyst of the third ventricle. The inquest was held soon after my report was submitted and the circumstance of death was stated as natural.

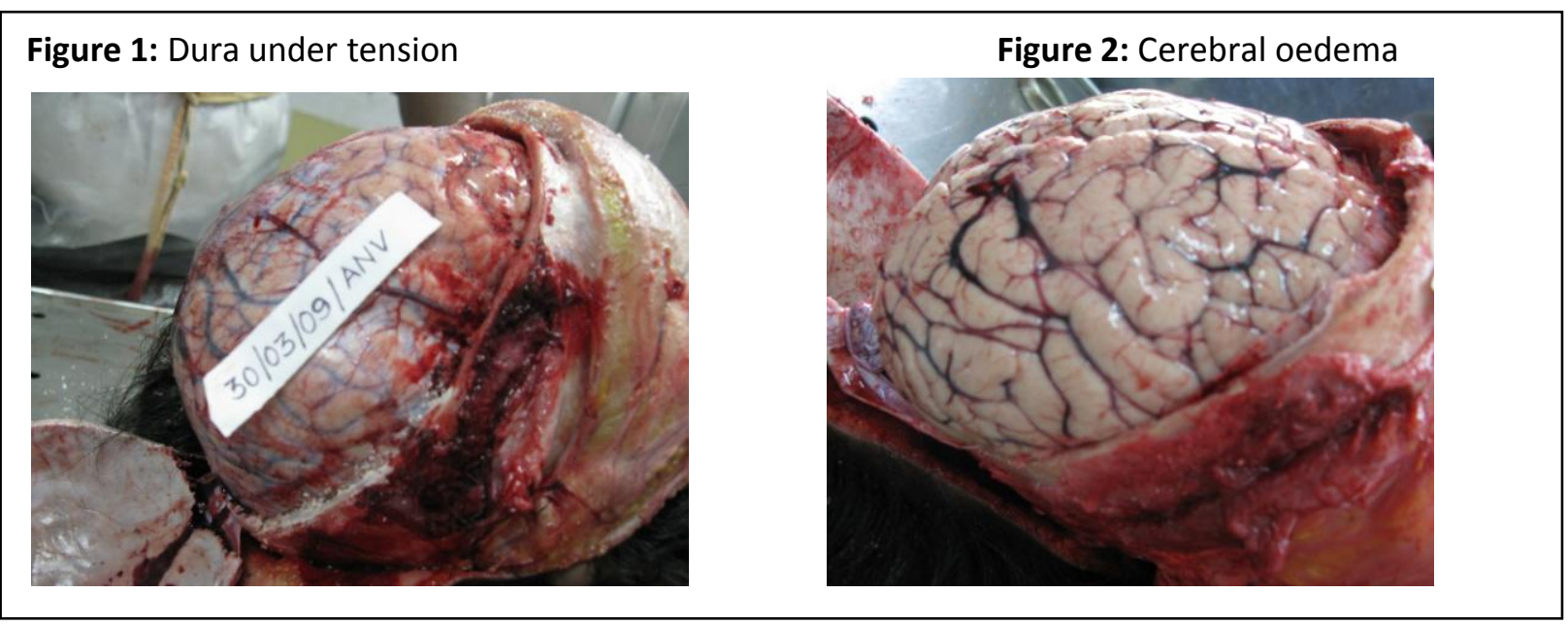

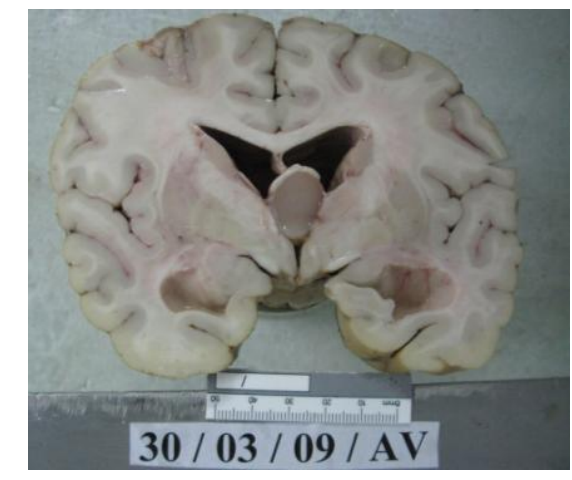

Figure 3

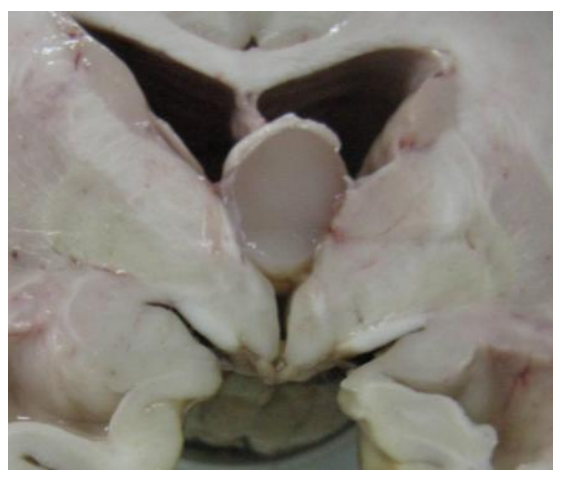

Figure 4
Figure 3 : Coronal section of the brain showing the colloid cyst in the $3^{\text {rd }}$ ventricle and dilated lateral ventricles

Figure 4: Close up view of colloid cyst

\section{Discussion}

Colloid cyst in the third ventricle has a rare occurrence world wide. The lesion classically presents in the $2^{\text {nd }}$ to $4^{\text {th }}$ decades of life but is rarely found in children with only $1-2 \%$ in patients younger than 10 years[2]. Ultrastructure and immunohistochemistry supports origin from displaced endodermal tissue. [3][4] A familial predisposition has also been reported[5].The colloid cyst has a delicate thin fibrous capsule filled with gelatinous mucoid substance. They can range in size from 3 to $40 \mathrm{~mm}$. The lining epithelium may be of mucus secreting cuboidal, psuedostratified or columnar[6]. Cyst contents are PAS positive. Although benign there is malignant transformation reported in the literature[7]. 
The close proximity of the cyst to the foramen of Monro and its' relative mobility causes it to obstruct the CSF flow transiently, leading to hydrocephalus (ball valve effect)[8][9]. This presents as intermittent headache as in our case, which may change with head posture. Occasionally patients will complain of vertigo, memory loss and diplopia. Some patients seldom present with symptoms. However, complete obstruction of the foramen leading to hydrocephalus may result in sudden death as in our case since the cyst was large (sudden death has not been reported in cysts less than 1 $\mathrm{cm}[10]$. Though it was not found in this case, haemorrhage in to the cyst has also been reported as a cause of sudden death[11].

The pulmonary oedema in the above case can be explained by the increase of extravascular lung water in sustained neurological damage[12] (which is a well known component of Virchow's triad in increased intracranial pressure.

As in our case most of the patients will have a long standing episodic headache which may be mild and relieved by over the counter simple analgesics. Rapidly worsening or frequent unexplained headaches should urge the need for further investigations including Computed Tomography or Magnetic Resonance Imaging which will be diagnostic of a colloid cyst. Depending on the size of the cyst, endoscopic and microsurgical resection is curative and carries a low risk.

\section{Conclusion}

This case report shows that although rare it is important for both the Clinician and the Forensic Pathologist to include this entity in the differential diagnosis of headaches in children and in cerebral oedema at autopsy. Demonstration of the colloid cyst is difficult as it is easily missed in dissection which signifies the importance of brain fixation.

\section{References}

1. Hernesniemi J, Leivo S. Management outcome in third ventricular colloid cysts in a defined population: a series of 40 patients treated mainly by transcallosal microsurgery. Surgical Neurology 1996; 45: 2-14.

2. Suh DY, Mapstone T. Pediatric supratentorial intraventricular tumours. Neurosurgical Focus 2001 Jun; 10(6):E4.

3. Ho KL, Garcia JH. Colloid cyst of the third ventricle. Ultrastructural features are compatible with endodermal derivation. Acta Neuropathologica 1992; 83: 605-612.

4. Shibata T, Burger PC. Origin of colloid cyst: immunoperoxidase study. No To Shinkei 1987 Oct; 39(10): 953-958.

5. Joshi SM, Gnanalingham KK, Mohaghegh P, Wilson A, Elsmore A. A case of familial third ventricular colloid cyst. Emergency Medicine Journal 2005 Dec; 22(12): 909910.

6. Shaktawat SS, Salman WD, Twaij Z, AlDawoud A. Unexpected death after headache due to a colloid cyst of the third ventricle. World Journal of Surgical Oncology 2006; 4: 47.

7. Sahara Y, Nagasaka T, Takayasu M, Takagi T, Hata N, Yoshida J. Recurrence of a neurenteric cyst with malignant transformation in the foramen magnum after total resection. Case report. Journal of Neurosurgery 2001; 95: 341-345.

8. Dolinak D, Matshes EW, Lew EO. Central nervous system disease. Forensic pathology principles and practice. Elsevier. 2005; 101

9. Smith C. Non traumatic neurological conditions in medicolegal work. Forensic neuropathology. Edward Arnold. 2005; 161

10. Hamlat A, Pasqualini E, Askar B. Hypothesis about the physiopathology of acute deterioration and sudden death caused by colloid cysts of the third ventricle. Medical hypotheses 2004. 63(6):1014-1017. 
11. Beems T, Menovsky $T$, Lammens $M$. Haemorrhagic colloid cyst: Case report and review of the literature. Surgical Neurology 2006 Jan; 65(1):84-86.

12. Smith WS, Matthay MA, Evidence for a hydrostatic mechanism of human neurogenic pulmonary oedema. Chest 1997; 111: $1326-1333$.

\section{Contribution of authors}

Performing the autopsy-ANV

Opinion- ANV

Writing the manuscript -ANV,CDW

Revising the manuscript- ANV,CDW

\section{Picture story- Facial injuries in females}

PAS Edirisinghe, Department of Forensic Medicine, Faculty of Medicine, University of Kelaniya
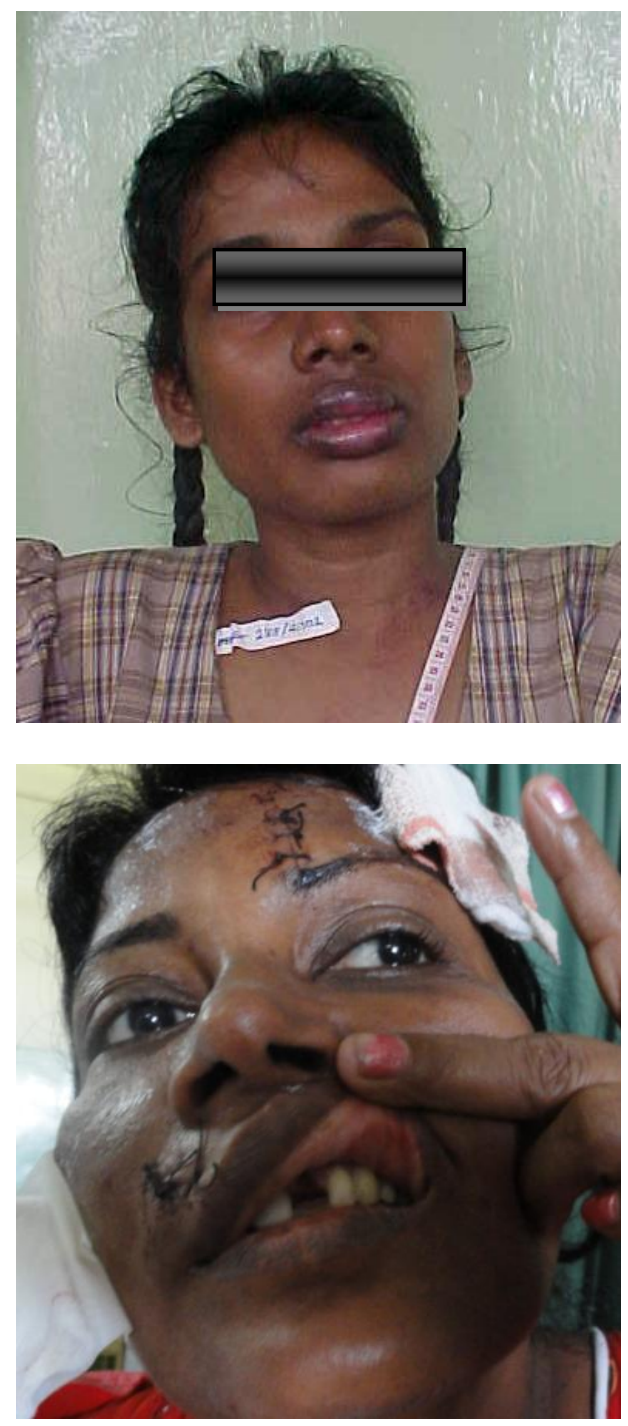

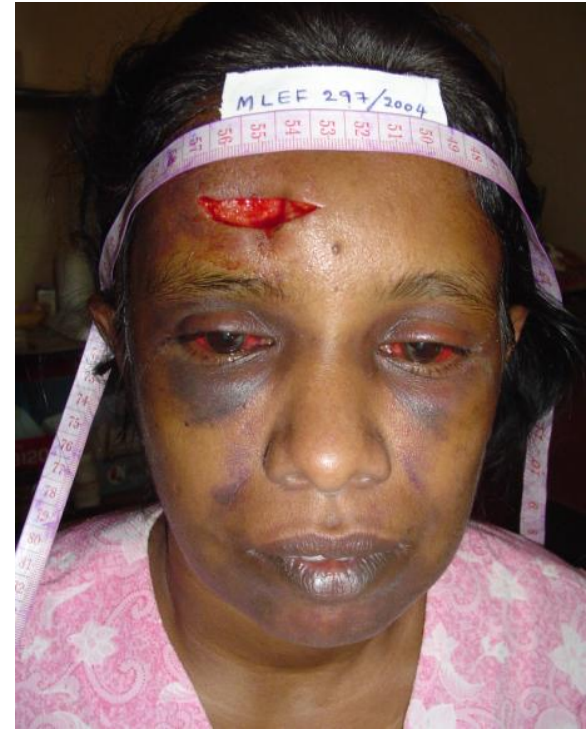

A doctor finding injuries on the face of a female has to suspect domestic violence. Although in majority of females, these injuries individually or collectively are non grievous, grievous injuries are not uncommon to find. Blunt force injuries due to slaps or fist blows to mouth and eyes are common. However, occasional cut injuries may be encountered.

A detailed history of similar episodes over the years has to be taken to understand the depth of the problem. Research indicates that the mother's experiences of domestic violence have a direct effect on children's mental health. 